\title{
Takotsubo cardiomyopathy in amiodarone-induced hyperthyroidism
}

\author{
Ismael Capel, Elisabet Tasa-Vinyals, Albert Cano-Palomares, Irene Bergés-Raso, \\ Lara Albert, Mercedes Rigla and Assumpta Caixàs
}

Endocrinology Department, Parc Taulí Sabadell University Hospital, Sabadell Barcelona, Spain
Correspondence should be addressed to I Capel

Email

icapel@tauli.cat

\section{Summary}

Takotsubo cardiomyopathy (TC) is an atypical, severe but reversible form of acute heart insufficiency. It typically presents with left ventricular failure, transient apical and mid-segments hypokinesis, absence of significant coronary stenosis and new electrographic abnormalities and/or elevation in serum cardiac enzymes. Although TC ('broken heart syndrome') has classically been associated with emotional trauma, evidence suggests that other precipitants might exist, including iatrogenic and thyroid-mediated forms. Thyroid disease is a relatively common comorbidity in TC patients. We report a case of TC in a postmenopausal female with no history of emotional trauma or other potential precipitant factors who was diagnosed with amiodarone-induced hyperthyroidism during her hospital stay. Though some case reports of thyroidrelated TC exist, we are not aware of any other reported case of TC precipitated by amiodarone-induced hyperthyroidism.

\section{Learning points:}

- TC is a relatively new, rare, transient, severe, but reversible cardiovascular condition that is characterized by an acute left ventricular cardiac failure, which can clinically, analytically and electrocardiographically mimic an acute myocardial infarction.

- Many precipitant factors have been described in TC, being the most classical and emotional trauma. However, thyroid dysfunction is also a significant condition frequently found in patients with TC.

- A hypercatecholaminergic state leading to cardiomyocyte damage has been established as the main fact of TC physiopathology. Hyperthyroidism induces an upregulation of $\beta$-adrenergic receptors.

- Both hyperthyroidism and hypothyroidism have been related with TC development. Most reported cases of TC involving thyroid dysfunction correspond to hyperthyroidism due to Graves-Basedow disease, but there are also descriptions with severe hypothyroidism, radioiodine treatment or thyroid surgery.

- Amiodarone is a class III antiarrhythmic agent widely used, and it is a well-known cause of thyroid dysfunction, which can present either with hypothyroidism or hyperthyroidism, as approximately 40 percent of the amiodarone molecule is composed of iodine.

- In this case, a type II amiodarone-induced hyperthyroidism was the precipitant factor of a TC in a patient with a pre-existing atrial fibrillation. Given the high prevalence of atrial fibrillation and the wide use of amiodarone, the risk of this iatrogenic effect should be taken into account.

\section{Background}

Takotsubo syndrome (TC) also known as transient apical ballooning syndrome, left ventricular apical ballooning syndrome or the more poetic broken heart syndrome is a relatively new, rare, transient, severe, but reversible cardiovascular condition in which clinical and research interest has been growing recently $(1,2)$. It is characterized

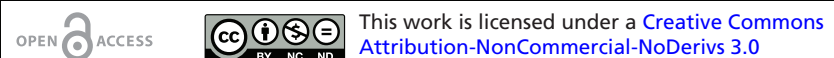
Unported License.
}

(c) 2017 The authors
http://www.edmcasereports.com
Published by Bioscientifica Ltd 
by an acute left ventricular cardiac failure which can clinically, analytically and electrocardiographically mimic an acute myocardial infarction. Its hallmark features are an anodyne coronarography, with no evidence of stenosis or obstruction of the coronary arteries, and characteristic ventriculographic findings, which reveal a characteristic pattern of left ventricular dyskinesis. First described in 1991 in Japan (3), TC owes its name to a Japanese device used in traditional octopus fishing, the takotsubo, the shape of which is characteristic of the ventriculographic images found in these patients.

An international collaborative systematic review including more than 1100 patients that examined the most frequent comorbidities in TC patients concluded that thyroid disease is among them: the study found that TC patients had a relatively high prevalence of psychological disorders, pulmonary diseases, malignancy, neurologic diseases, chronic kidney disease and thyroid diseases (4). Moreover, hyperthyroidism - and particularly thyrotoxicosis - is a well-known cause of cardiac complications.

Though thyroid-related agents such as levothyroxine and radioiodine therapy for toxic multinodular goiter have been linked to TC (5), as far as we are aware, the literature does not currently include amiodarone, among the commonly described iatrogenic causes of TC. Amiodarone is a class III antiarrhythmic agent used to treat various types of cardiac arrhythmias, both ventricular and atrial. It is commonly prescribed in patients suffering from atrial fibrillation, which is commonly diagnosed in elderly people. Amiodarone is a well-known cause of thyroid dysfunction, which can present either with hypothyroidism or hyperthyroidism, as approximately 40 percent of the amiodarone molecule is composed of iodine that can interact with the thyroid gland. Amiodarone-induced hyperthyroidism is classified in type I, type II or mixed states. Type I amiodarone-induced hyperthyroidism usually occurs in patients with preexisting thyroid pathology, usually multinodular goiter or latent Graves' disease, and is caused by an increase in the synthesis and secretion of thyroid hormones; conversely, type II amiodarone-induced hyperthyroidism occurs in previously healthy thyroid glands and is a consequence of destructive thyroiditis, which causes stored thyroid hormone to be abruptly released into the bloodstream. Although type II hyperthyroidism is usually transitory, type I tends to persist after the administration of amiodarone is suspended.

\section{Case presentation}

A 79-year-old female was admitted to our hospital presenting with dyspnea and palpitations, not associated with chest pain, present for several hours. Her medical history included hypertension, type 2 diabetes mellitus, atrial fibrillation, colonic diverticulosis, depressive syndrome, osteoporosis and mastectomy due to breast cancer three years prior to admission. Her history of cardiovascular disease included a single episode of ischemic cardiomyopathy two years before admission, when she had been diagnosed with a singlevase (anterior descendant artery) acute myocardial infarction, successfully re-vascularized with percutaneous angioplasty, after which the remaining left ventricle ejection fraction (LVEF) was 64 percent. Barthel Index was 85. The patient was receiving the following medication: omeprazole $20 \mathrm{mg}$ q.d., metformin $850 \mathrm{mg}$ b.i.d., calcifediol $266 \mu$ g q. 1 month, calcium 500 mg q.d., alendronate $70 \mathrm{mg} \mathrm{q}$. 1 week, acenocumarol $4 \mathrm{mg}$ (periodic dosing), amiodarone $200 \mathrm{mg}$ q.d., torasemide $5 \mathrm{mg}$ q.d., enalapril $5 \mathrm{mg}$ q.d., letrozole $2.5 \mathrm{mg}$ q.d., citalopram $10 \mathrm{mg}$ q.d., acetaminophen on demand (up to $3 \mathrm{~g}$ q.d.) and lormetazepam on demand up to $2 \mathrm{mg}$ q.d.

Though conscious and orientated, the patient was pale and diaphoretic, slightly hypothermic at $35.6^{\circ} \mathrm{C}$ and presented tachycardia $(146 / \mathrm{min})$ despite not being hypotense $(156 / 108 \mathrm{mmHg})$ nor anoxic $\left(\mathrm{SpO}_{2} 96\right.$ percent). Cardiac auscultation revealed arrhythmic tones with an aortic murmur II/VI, chronic venous insufficiency signs and no edemas. Respiratory auscultation revealed hypophonesis with some crackles on the right pulmonary base. After a few hours in the emergency department, the patient developed cardiac insufficiency and was electrocardiographically found to be in atrial fibrillation with a fast ventricular response (150/min).

Diuretics, vasodilators and digoxin were administered.

\section{Investigation}

Echocardiogram showed apical and mid-segment akinesia, with hypokinesis in basal segments. Myocardial damage was objectivized with elevated cardiac troponin, accompanied by a slight leucocytosis and increased inflammatory markers. Thoracic radiography showed a pattern of vascular redistribution, cardiomegaly and signs of pulmonary edema. Coronary angiography did not demonstrate any stenotic or spasmodic changes when 
compared to previous data, which already showed a stent stenosis of approximately 30 percent. Ventriculography was compatible with apical dyskinesia, with the classical Takotsubian apical ballooning and a preserved LVEF. The patient was admitted to the cardiology hospitalization unit with her usual treatment plus transdermal nitrates and digoxin. Periodic controls showed a decrease in myocardial damage makers and electrocardiographic stability with remaining normofrequent atrial fibrillation.

Five days after initial hospital admission, a routine analytical control objectivized hyperthyroidism: thyrotropin (TSH), $0.014 \mu \mathrm{U} / \mathrm{mL}$ (reference range (RR): 0.4-4.0) and free thyroxine $\left(\mathrm{FT}_{4}\right), 4.48 \mathrm{ng} / \mathrm{dL}$ (RR: $0.8-1.8$ ). The patient was asymptomatic and had no history of personal or family thyroid disease. She was physically re-examined and no goiter, exophthalmos or pretibial myxedema was found. Antibody study was negative, with thyroid-stimulating immunoglobulin (TSI): $<0.9 \mathrm{mUI} / \mathrm{mL}$ (reference value (RV): <2), anti-thyroperoxidase: $6.16 \mathrm{UI} / \mathrm{mL}$ (RV: <15), anti-thyroglobulin $14.88 \mathrm{UI} / \mathrm{mL}$ (RV: <100). Exogenous sources of iodine were investigated and discarded except for amiodarone, an antiarrhythmic drug she had been receiving for the past two months with doses of $200 \mathrm{mg}$ every $24 \mathrm{~h}$. A diagnosis of type I amiodarone-induced hyperthyroidism was first suspected, and this drug was suspended.

\section{Treatment}

She was empirically treated with antithyroidal drugs (methimazole $10 \mathrm{mg}$ q. $8 \mathrm{~h}$ ), which proved ineffective to control thyroid function: thyrotropin (TSH): $<0.014 \mu \mathrm{U} / \mathrm{mL}$, free thyroxine $\left(\mathrm{FT}_{4}\right): 7.55 \mathrm{ng} / \mathrm{dL}$ and free triiodothyronine $\left(\mathrm{FT}_{3}\right): 4.04 \mathrm{pg} / \mathrm{mL}$ (RR: 3.0-6.9). The patient nevertheless remained asymptomatic. The new diagnostic hypothesis was type II amiodarone-induced hyperthyroidism, for which prednisone $45 \mathrm{mg}$ q.d. was added to the previous treatment with a good response: thyrotropin (TSH): $<0.014 \mu \mathrm{U} / \mathrm{mL}$; free thyroxine $\left(\mathrm{T}_{4}\right)$ : $3.9 \mathrm{ng} / \mathrm{dL}$ and free triiodothyronine $\left(\mathrm{T}_{3}\right): 1.65 \mathrm{pg} / \mathrm{mL}$. The patient remained asymptomatic at all times.

\section{Outcome and follow-up}

A few days after the hyperthyroid state was detected, methimazole had to be stopped due to the need for urgent abdominal surgery (ischemic colitis with perforation requiring right hemicolectomy), a circumstance that was most likely unrelated to the TC or the hyperthyroidism.
Descendant doses of corticoids were maintained during the patient's stay at the intensive care unit until the thyroid function was totally normalized. After six weeks in the hospital, the patient was discharged with normal thyroid function and a complete resolution of the TC, with cardiovascular symptoms and signs back to the patient's baseline situation.

Unfortunately, three months later, patient developed a new episode of ischemic colitis with bowel perforation and generalized sepsis and fatal outcome. Thyroid function was assessed again during this second hospital stay, and it remained normal.

\section{Discussion}

TC has been linked with many thyroid disorders, the most frequent being hyperthyroidism due to Graves-Basedow disease, but there are also reports in patients with hypothyroidism, euthyroid syndrome, thyroid surgery or thyroid cancer $(6,7)$.

When reviewing TC physiopathology, most authors acknowledge that hypercatecholaminergic states lead to cardiomyocyte damage via calcium overload, which can be histologically observed in the form of contraction band necrosis and other typical TC anatomopathological findings. However, it has been hypothesized that hyperthyroid states clinically, physiologically and biochemically mimic hyperadrenergic states, and/or interact with those in a way that creates synergic pathways to cardiac overload and failure. Thyroid hormones have been described to have direct and indirect effects on cardiomyocytes via complex mechanisms that likely involve the upregulation of $\beta$-adrenergic receptors (8). Regarding hypothyroid states, though they appear to relate to TC far less often than do hyperthyroid states, it has been suggested that they may have a link with TC in and of themselves, irrespective of the thyroid profile's restoration or inversion with the levothyroxine replacement. Hypothyroid patients may present alterations of the autonomic nervous system regardless of their thyroid status at a given time. Hypothyroidism could also induce coronary artery spasm and a decrease in coronary reserve, hypocontractility, cardiac atrophy and dilatation. Another suggested mechanism in those few cases where hypothyroidism has led to TC involves the development of a ventricular thrombus, a phenomenon that has been described in rare cases of left ventricular systolic dysfunction such as TC. In such cases, the systolic dysfunction is associated with cardioembolic adverse events, such as strokes (9). 
Here, we present a case of TC in a patient with amiodarone-induced hyperthyroidism. In our opinion, the plausible mechanism of the heart dysfunction in this case would be the high levels of thyroid hormones and not a direct effect of the drug. Although we may think that here amiodarone has only an anecdotal role in causing hyperthyroidism, we believe it is important to emphasize the cause of thyroid dysfunction because it is a widely used antiarrhythmic drug in patients with underlying heart disease, in which a TC would be particularly problematic.

Our search has failed to identify any studies specifically associating TC and amiodaroneinduced thyroid disorders in any of its presentations (hyperthyroid or hypothyroid forms). We have identified some case studies reporting the need for amiodarone administration after arrhythmic complications of TC (10). The most common thyroid-related iatrogenic agent associated with TC that we have identified among the studies retrieved by our search is thyroxine, usually in its therapeutic use as levothyroxine for the treatment of hypothyroidism but also in non-medical uses, such as self-administration of porcine thyroxine purchased on the internet $(5,6)$.

In conclusion, we can say that thyroid disorders are a frequent condition in patients with $\mathrm{TC}$, and that amiodarone, a well-known thyroid dysfunction causing agent, can be involved in the development of TC, an especially severe problem if it presents in a patient with underlying heart disease.

\section{Declaration of interest}

The authors declare that there is no conflict of interest that could be perceived as prejudicing the impartiality of the research reported.

\section{Funding}

This research did not receive any specific grant from any funding agency in the public, commercial or not-for-profit sector.

\section{Patient consent}

A written informed consent was not obtained from the patient as the patient is dead. The patient's identity remained anonymous in this report.

\section{Author contribution statement}

I Capel, I Bergés-Raso, L Albert and A Caixàs were involved in the patient care, reviewed the literature and prepared the manuscript. E Tasa-Vinyals, A Cano-Palomares and M Rigla reviewed the literature and prepared the manuscript.

\section{References}

1 Pellicia F, Greco C, Vitale C, Rosano G, Gaudio C \& Kaski JC 2014 Takotsubo syndrome (stress cardiomyopathy): an intriguing clinical condition in search of its identity. American Journal of Medicine $\mathbf{1 2 7}$ 699-704. (doi:10.1016/j.amjmed.2014.04.004)

2 Yoshikawa T 2015 Takotsubo cardiomyopathy, a new concept of cardiomyopathy: clinical features and pathophysiology. International Journal of Cardiology 182 297-303. (doi:10.1016/j.ijcard.2014.12.116)

3 Dote K, Sato H, Tateishi H, Uchida T \& Ishihara M 1991 Myocardial stunning due to simultaneous multivessel coronary spasms: a review of 5 cases. Journal of Cardiology 21 203-214.

4 Pelliccia F, Parodi G, Greco C, Antoniucci D, Brenner R, Bossone E, Cacciotti L, Capucci A, Citro R, Delmas C, et al. 2015 Comorbidities frequency in Takotsubo syndrome: an international collaborative systematic review including 1109 patients. American Journal of Medicine 128 654.e11-654.e19. (doi:10.1016/j.amjmed.2015.01.016)

5 Izumi Y 2013 Drug-induced Takotsubo cardiomyopathy. Heart Failure Clinics 9 225-231. (doi:10.1016/j.hfc.2012.12.004)

6 Aggarwal S, Papani R \& Gupta V 2015 Can thyroid break your heart? Role of thyroid in Takotsubo cardiomyopathy: a single center retrospective study. International Journal of Cardiology 184 545-546. (doi:10.1016/j.ijcard.2015.02.058)

7 Lee SJ, Kang JG, Ryu OH, Kim CS, Ihm SH, Choi MG, Yoo HJ \& Hong KS 2009 The relationship of thyroid hormone status with myocardial function in stress cardiomyopathy. European Journal of Endocrinology 160 799-806. (doi:10.1530/EJE-08-0808)

8 Eliades M, El-Maouche D, Choudhary C, Zinsmeister B \& Burman KD 2014 Takotsubo cardiomyopathy associated with thyrotoxicosis: a case report and review of the literature. Thyroid $\mathbf{2 4} 383-389$. (doi:10.1089/thy.2012.0384)

9 Micallef T, Gruppetta M, Cassar A \& Fava S 2011 Takotsubo cardiomyopathy and severe hypothyroidism. Journal of Cardiovascular Medicine 12 824-827. (doi:10.2459/JCM.0b013e3283403454)

10 Al-Salameh A, Allain J, Meimoun P, Benali T \& Desailloud R 2014 Takotsubo cardiomyopathy can occur in patients with apathetic hyperthyroidism. Thyroid 24 400-401. (doi:10.1089/thy.2013.0354)

Received in final form 21 December 2016

Accepted 23 January 2017 